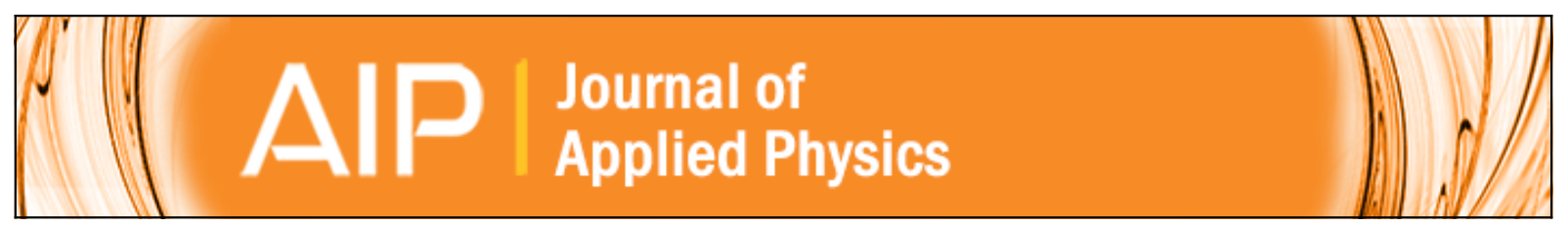

\title{
Theoretical analysis of the intrinsic magnetic noise spectral density of magnetostrictive-piezoelectric laminated composites
}

X. Zhuang, C. Cordier, S. Saez, M. Lam Chok Sing, C. Dolabdjian, J. Gao, J. F. Li, and D. Viehland

Citation: Journal of Applied Physics 109, 124512 (2011); doi: 10.1063/1.3594714

View online: http://dx.doi.org/10.1063/1.3594714

View Table of Contents: http://scitation.aip.org/content/aip/journal/jap/109/12?ver=pdfcov

Published by the AIP Publishing

\section{Articles you may be interested in}

Direct current force sensing device based on compressive spring, permanent magnet, and coil-wound magnetostrictive/piezoelectric laminate

Rev. Sci. Instrum. 84, 125003 (2013); 10.1063/1.4838615

Eddy-current effect on resonant magnetoelectric coupling in magnetostrictive-piezoelectric laminated composites J. Appl. Phys. 114, 027010 (2013); 10.1063/1.4812218

Magnetoelectric effect in magnetostrictive/piezoelectric laminate composite Terfenol- D/Li Nb O 3 [ ( z x t w ) $129^{\circ} / 30^{\circ}$ ]

Appl. Phys. Lett. 88, 172903 (2006); 10.1063/1.2198486

Push-pull mode magnetostrictive/piezoelectric laminate composite with an enhanced magnetoelectric voltage coefficient

Appl. Phys. Lett. 87, 062502 (2005); 10.1063/1.2007868

Circumferentially magnetized and circumferentially polarized magnetostrictive/piezoelectric laminated rings

J. Appl. Phys. 96, 3382 (2004); 10.1063/1.1781764

MIT LINCOLN

LABORATORY CAREERS

Discover the satisfaction of innovation and service

to the nation
- Space Control

- Air \& Missile Defense

- Communications Systems \& Cyber Security

- Intelligence, Surveillance and

Reconnaissance Systems

- Advanced
Electronics
- Tactical Systems
- Homeland
Protection
- Air Traffic Control

LINCOLN LABORATORY

MassachusetTs Institute of TeChNOLOgY

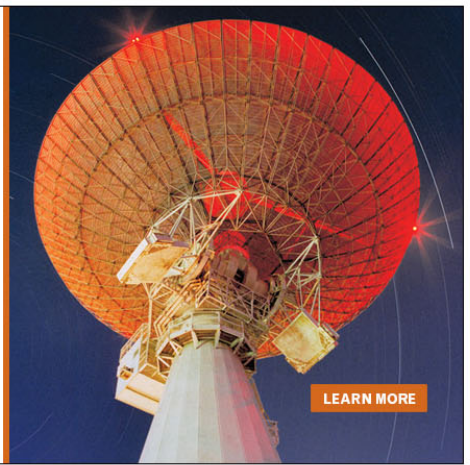




\title{
Theoretical analysis of the intrinsic magnetic noise spectral density of magnetostrictive-piezoelectric laminated composites
}

\author{
X. Zhuang, ${ }^{1}$ C. Cordier, ${ }^{1}$ S. Saez, ${ }^{1}$ M. Lam Chok Sing, ${ }^{1}$ C. Dolabdjian, ${ }^{1, a)}$ J. Gao, ${ }^{2}$ J. F. Li, ${ }^{2}$ \\ and D. Viehland ${ }^{2}$ \\ ${ }^{1}$ Groupe de Recherche en Informatique, Image, Automatique et Instrumentation de Caen (GREYC), \\ CNRS UMR 6072-ENSICAEN and Université de Caen Basse Normandie, France 14050 Caen Cedex \\ ${ }^{2}$ Department of Materials Science and Engineering, Virginia Tech, Blacksburg, Virginia 24061, USA
}

(Received 25 February 2011; accepted 25 April 2011; published online 28 June 2011)

\begin{abstract}
The equivalent magnetic noise spectral density level for long type magnetostrictive-piezoelectric laminated composites has been investigated by using a 1D equivalent circuit model, and exemplified for a longitudinal-transverse mode. The theoretical developments explain well our experimental results. The findings show that similar ultimate magnetic noise spectral noise density can be expected whether using either charge or voltage amplifier detection methods. The findings show that a volume effect and the dielectric loss factor of the piezoelectric layer are the dominant sources of the noise floor. Our findings show that the noise scales as $1 / \sqrt{ } f$. The lowest equivalent noise floor value that has been observed is $10 / \sqrt{ } f \mathrm{pT} / \sqrt{ } \mathrm{Hz}$ for $f \ll 10 \mathrm{kHz}$, with a white noise level of $100 \mathrm{fT} / \sqrt{ } \mathrm{Hz}$ above $10 \mathrm{kHz}$. (C) 2011 American Institute of Physics.
\end{abstract}

[doi:10.1063/1.3594714]

\section{INTRODUCTION}

Magneto-electric (ME) laminated composites have excellent performances as sensors for magnetic field detection. ${ }^{1-3}$ The magnetic signal is converted into an electrical signal via elastic strain or stress interactions between magnetostrictive and piezoelectric layers. Hence, a strong current or voltage coupling is realized as a product of the magnetostrictive-piezoelectric properties. ${ }^{4-6}$ Stresses, or displacements, in the magnetic or piezoelectric layers convert energy between the layers of the laminate. These two elastic elements have been regarded as mechanical voltage and current sources respectively, in our prior equivalent-circuit models. ${ }^{7,8}$ The ME laminated composites can be considered as voltage or charge sources, ${ }^{9}$ with associated ME voltage $\alpha_{M E}^{V}$ or charge $\alpha_{M E}^{Q}$ coefficients. Both are key factors for the signal transfer capabilities of ME laminated composites. ${ }^{7,10}$

The equivalent magnetic noise spectral density is the best measure of the sensitivity of magnetic sensors to small magnetic field variations: ${ }^{11}$ the lower the noise level, the better the sensor resolution. In order to amplify a signal sensed by an ME laminate, a charge or voltage amplifier is required. ${ }^{12}$ Both detection methods have associated noise sources. For ME laminated composites and amplifiers, the noise sources can be classified as: (i) the dielectric loss in the piezoelectric layers, (ii) the Johnson noise of the resistors, and (iii) the noise sources of the operational amplifier used. ${ }^{10,12,13}$ The intrinsic sensor noise sources determine the ultimate noise level which is the case when the other noise sources can be considered as negligible. We neglect, in this model, the noise of the magnetostrictive layers, because we expect the latter to induce a much smaller noise contribution compared to the intrinsic piezoelectric material noise.

\footnotetext{
${ }^{\text {a) }}$ Author to whom correspondence should be addressed. Electronic mail: christophe.dolabdjian@unicaen.fr
}

Around a certain dc magnetic bias field the response of $\mathrm{ME}$ composites for a small AC magnetic signal is linear. ${ }^{14-17}$ So, we have used this assumption for our modeling. On the basis of the linear piezoelectric and magnetostrictive constitutive equations, an ME laminated sensor can be represented by an equivalent circuit, ${ }^{7,18,19}$ where the magneto-elastic coupling and elasto-electric coefficients are mimicked by an ideal transformer. Furthermore, the voltage or charge coefficients can be determined using the piezoelectric, magnetostrictive, elastic compliance, and permeability constants, and the relative volume factor of layers. Using this equivalent circuit and by taking account of all noise sources, we have evaluated the ultimate equivalent magnetic noise floor of $\mathrm{ME}$ sensors.

In the following sections, we shall analyze the theoretical noise limits of a ME sensor. The noise sources will be described using both charge and voltage amplifiers, yielding the ultimate and intrinsic magnetic noise of the sensor. The given theoretical model is generalized for all ME sensor operational modes. Experimental results and measurement conditions will also be presented and compared to the model.

\section{THEORETICAL ANALYSIS}

\section{A. 1D model at low frequencies}

The key importance of the 1D approximation method as given by Mason's model is that it helps to evaluate the performance and the working principles of an ME sensor. $^{7,18,20,21}$ Notice that the mechanical motions are considered to exist only in the longitudinal direction of the sensor. For the perpendicular direction, magnetostrictive and piezoelectric layers are considered as free bodies. Therefore, there are no perpendicular strains or stresses between the layers in given model. There are four basic operational modes that need to be considered for 1D ME laminated 


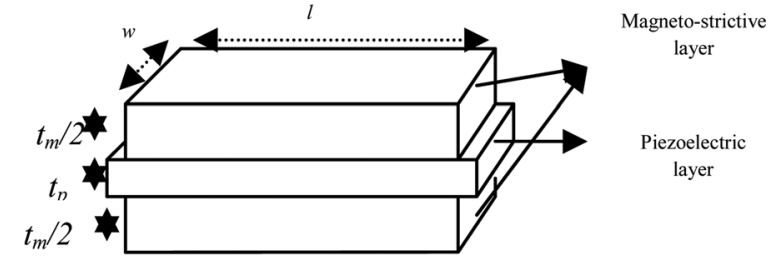

$\overbrace{}^{\wedge} \boldsymbol{\varphi}$
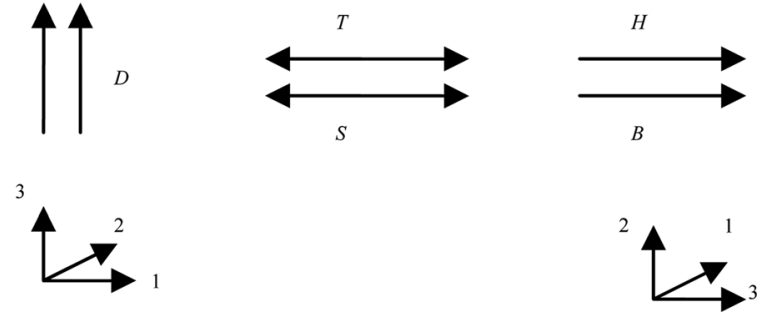

FIG. 1. Sketch view of the laminated magnetoelectric sensor model.

sensor modeling. ${ }^{7,18}$ These are classified by the direction of the sensed magnetic field and polarized electric field. The "L-L" mode defines longitudinal magnetic field and longitudinal polarized electric field axes directed along the long axes of the laminate. Similarly, the "L-T," "T-L," and "T-T" modes designate longitudinal magnetic and transverse poled electric fields, transverse magnetic and longitudinal electric fields, and transverse magnetic and transversely poled electric fields, respectively. In 1D, the displacement direction is along the longitudinal or long axis of the laminate sensor.

For example, a schematic of a laminate with a L-T configuration consisting of two magnetostrictive layers and one piezoelectric layer is given in Fig. 1. In this example, the parameters $E$ (electric field), $D$ (electric displacement), $H$ (magnetic field), and $B$ (induction field) are shown along the 3 direction of their self-axes. The dimensions of the piezoelectric and magnetostrictive layers are $l \times w \times t_{p}$ and $l \times w \times t_{m}$, respectively. The forces at both edges, $F_{1}$ and $F_{2}$, of the ME laminated sensor are given by

$$
\left\{\begin{array}{l}
F_{1}=F_{1, m}+F_{1, p} \\
F_{2}=F_{2, m}+F_{2, p}
\end{array}\right.
$$

In free boundary conditions, for the type of sensors we tested, these forces are null $\left(F_{1}=F_{2}=0\right)$. From the linear basic piezoelectric and magnetostrictive equations,

$$
\left\{\begin{array} { c } 
{ E _ { 3 } = \beta _ { 3 3 } ^ { T } D _ { 3 } - g _ { 3 1 , p } T _ { 1 } } \\
{ S _ { 1 } = s _ { 1 1 , p } ^ { D } T _ { 1 } + g _ { 3 1 , p } D _ { 3 } }
\end{array} \text { and } \left\{\begin{array}{c}
S_{1}=s_{33, m}^{H} T_{1}+d_{33, m} H_{3} \\
B_{3}=\mu_{33}^{T} H_{3}+d_{33, m} T_{1}
\end{array},\right.\right.
$$

we can obtain the following system of equations

$$
\left\{\begin{array}{l}
F_{1}=\left(\zeta_{p}+\zeta_{m}\right)\left(j \operatorname{tg} \frac{k l}{2} v_{1}+\frac{\left(v_{1}+v_{2}\right)}{j \sin k l}\right)-\frac{g_{31, p}}{j \omega s_{11}^{D}} \frac{t_{p}}{l} I+\frac{d_{33, m} A_{m}}{s_{33, m}^{H}} H_{3} \\
F_{2}=\left(\zeta_{p}+\zeta_{m}\right)\left(j \operatorname{tg} \frac{k l}{2} v_{2}+\frac{\left(v_{1}+v_{2}\right)}{j \sin k l}\right)-\frac{g_{31, p}}{j \omega s_{11}^{D}} \frac{t_{p}}{l} I+\frac{d_{33, m} A_{m}}{s_{33, m}^{H}} H_{3}
\end{array} .\right.
$$

$\omega$ is the angular speed of the elastic wave; $\zeta_{p}$ and $\zeta_{m}$ are the characteristic impedances of the piezoelectric and magnetostrictive layers, respectively, with $\zeta_{m}=\frac{k_{m} A_{m}}{\omega S_{33, m}^{H}}$ and $\zeta_{p}=\frac{k_{p} A_{p}}{\omega s_{11, p}^{0}}$; $v_{1}$ and $v_{2}$ are the vibration speeds at both sensor edges, $k$ is the wave number; $l$ is the length of the layers, and $j$ is the imaginary number symbol; $g_{31, p}$ is the piezoelectric constant; $I$ is the induced electric current; $d_{33, m}$ is the magnetostrictive constant; $A_{m}$ is the side surface area of the magnetostrictive layer; $s_{33, m}^{H}$ is the elastic compliance coefficient of the magnetostrictive material under constant magnetic field and $H_{3}$ is the sensed magnetic field along the 3 axis.

We also need to consider the electric potential that is coupled with (1), which can be obtained as

$$
\left\{\begin{array}{l}
F_{1}=\left(\zeta_{p}+\zeta_{m}\right)\left(j \operatorname{tg} \frac{k l}{2} v_{1}+\frac{\left(v_{1}+v_{2}\right)}{j \sin k l}\right)-\frac{g_{31, p}}{j \omega s_{11}^{D}} \frac{t_{p}}{l} I+\frac{d_{33, m} A_{m}}{s_{33, m}^{H}} H_{3} \\
F_{2}=\left(\zeta_{p}+\zeta_{m}\right)\left(j \operatorname{tg} \frac{k l}{2} v_{2}+\frac{\left(v_{1}+v_{2}\right)}{j \sin k l}\right)-\frac{g_{31, p}}{j \omega s_{11}^{D}} \frac{t_{p}}{l} I+\frac{d_{33, m} A_{m}}{s_{33, m}^{H}} H_{3} \\
V=\frac{g_{31, p}}{j \omega s_{11}^{D}} \frac{t_{p}}{l}\left(v_{1}+v_{2}\right)-\frac{I}{j \omega} \frac{\beta_{33}^{S} t_{p}}{l w}
\end{array}\right.
$$

where $V$ is the induced voltage. Thus, the equivalent circuit can be deduced from the above equations, as shown in Fig. 2. The latter presents a complete 1D equivalent circuit for a ME laminate sensor. ${ }^{7,18,19}$ We notice that the magnetic field $H$, voltage $V$, or current $I$ are linked to the vibration speed. For the L-T mode, we can define four parameters: (i) $\varphi_{p}=w \frac{d_{33, p}}{s_{33, p}^{E}}$, which is the coupling coefficient of the piezoelectric layer; (ii) $\varphi_{m}=w t_{m} \frac{d_{33, m}}{s_{33, m}^{H}}$, which is the coupling coefficient of the magnetostrictive layer; (iii) $Z_{1, \text { mech }}=j\left(\zeta_{p}+\zeta_{m}\right) \operatorname{tg} \frac{\mathrm{kl}}{2}$ and $Z_{2, \text { mech }}=\left(\zeta_{p}+\zeta_{m}\right) \frac{1}{j \sin k l}$, which are the equivalent mechanical impedances and (iv) $C=\frac{l w}{\beta_{33}^{s} t_{p}}=\varepsilon_{33}^{S} \frac{l w}{t_{p}}$, which is the capacitance of the piezoelectric layer.

\section{B. 1D model at the resonant frequency}

At the resonant frequency, the equivalent circuit of Fig. 2 can be simplified to that given in Fig. 3. At low frequencies, the electrical charge and voltage responses of the

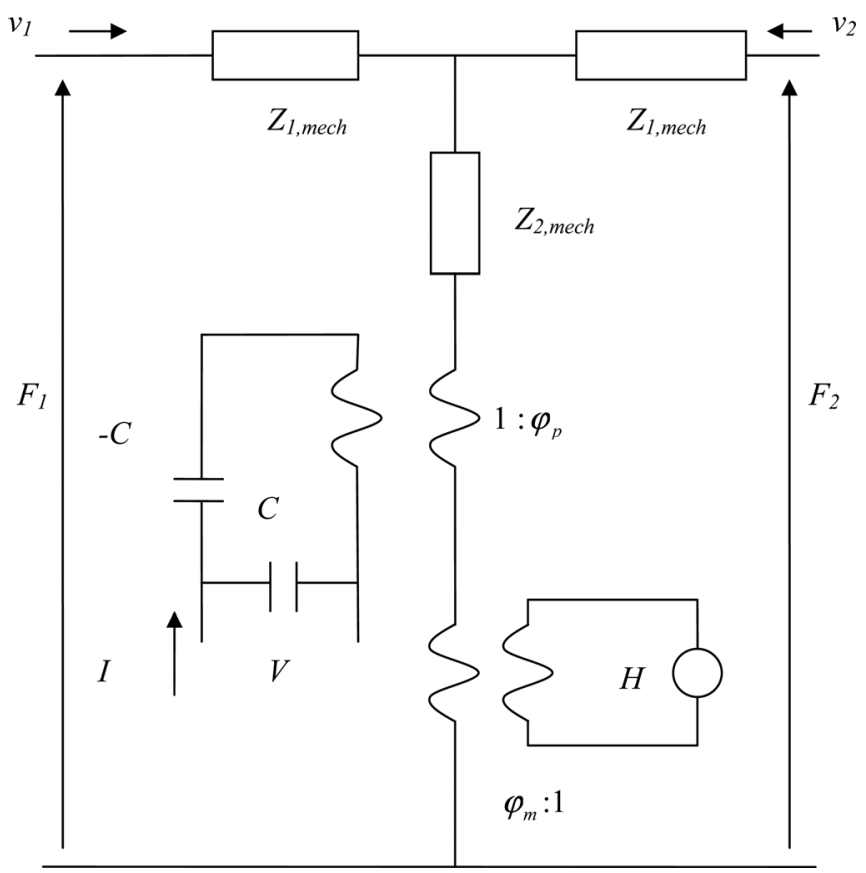

FIG. 2. Sketch view of the electrical 1D equivalent circuit of a magnetoelectric laminated sensor. 


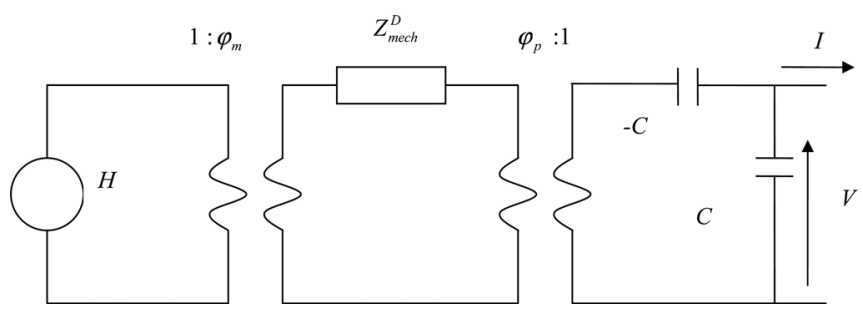

FIG. 3. Simplified equivalent circuit under free boundary conditions and at low frequency. $Z_{\text {mech }}^{D}\left(=Z_{1, \text { mech }} / / Z_{1, \text { mech }}+Z_{2, \text { mech }}\right)$ is the mechanical impedance of constant electric induction.

ME laminates are nearly constant with frequency. ${ }^{10}$ Consequently, the mechanical resistance can be written for the quasistatic state $\omega \ll 2 \pi f_{s}$ (where $f_{s}$ is the mechanical resonant frequency of the system) as

$$
Z_{\text {mech }}^{D}=Z_{1, \text { mech }} / / Z_{1, \text { mech }}+Z_{2, \text { mech }}=-j\left(\frac{A_{m}}{s_{33, m}^{H}}+\frac{A_{p}}{s_{11, p}^{D}}\right) \frac{1}{v k l} .
$$

We define $n$ as the thickness ratio of the magnetostrictive to piezoelectric layers of the sensor. This changes (3) to

$$
Z_{\text {mech }}^{D}=-j\left(\frac{n}{s_{33, m}^{H}}+\frac{1-n}{s_{11, p}^{D}}\right) \frac{A}{\omega l}
$$

where $A=w\left(t_{p}+t_{m}\right)$ is the side surface area of the sensor. Using the basic constitutive equations of $\left\{\begin{array}{c}S_{1}=s_{11, p}^{E} T_{1}+d_{31, p} E_{3} \\ D_{3}=\varepsilon_{33}^{T} E_{3}+d_{31, p} T_{1}\end{array}\right.$ and $\left\{\begin{array}{c}S_{1}=s_{33, m}^{H} T_{1}+d_{33, m} H_{3} \\ B_{3}=\mu_{33}^{T} H_{3}+d_{33, m} T_{1}\end{array}\right.$, we are then able to obtain the equivalent circuit model for the L-T mode of Fig. 3 to that in Fig. 4. The main difference in the circuits of these figures is that the capacitance, $-C$, is removed in Fig. 4. This is because we used the compliance coefficient $s_{11}^{E}$ at constant field instead of that at constant electric displacement $s_{11}^{D}$. Thus, we can obtain $Z_{\text {mech }}^{D}=-j\left(\frac{n}{s_{33, m}^{H}}+\frac{1-n}{s_{11, p}^{D}}\right) \frac{A}{\omega l}, \quad Z_{\text {mech }}^{E}=-j\left(\frac{n}{s_{33, m}^{H}}+\frac{1-n}{s_{11, p}^{E}}\right) \frac{A}{\omega l}, \quad$ and $j \omega C Z_{\text {mech }}^{D}=j \omega C Z_{\text {mech }}^{E}+\varphi_{p}^{2}$.

\section{Charge and voltage coefficients at low frequencies}

From the simplified equivalent circuits given in Figs. 3 and 4, the ME voltage coefficient (in $(\mathrm{V} / \mathrm{A}) / \mathrm{m}$ ) can be given as

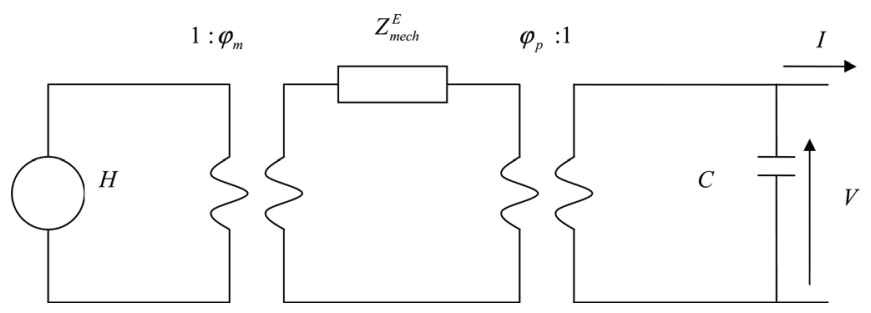

FIG. 4. Simplified equivalent circuit under free boundary conditions and at low frequency. $Z_{\text {mech }}^{E}\left(=Z_{1, \text { mech }} / / Z_{1, \text { mech }}+Z_{2, \text { mech }}\right)$ is the mechanical impedance of constant electric field.

$$
\alpha_{M E}^{V}=\left|\frac{d V}{d H}\right|=\left|\frac{\varphi_{m} \varphi_{p}}{\varphi_{p}^{2}+j \omega C_{0} Z_{\text {mech }}^{E}}\right|=\left|\frac{\varphi_{m} \varphi_{p}}{j \omega C Z_{\text {mech }}^{D}}\right|,
$$

and the ME charge coefficient (in $(\mathrm{C} / \mathrm{A}) / \mathrm{m})$ as

$$
\alpha_{M E}^{Q}=\left|\frac{d Q}{d H}\right|=\left|\frac{1}{j \omega} \frac{d I}{d H}\right|=\left|\frac{\varphi_{m} \varphi_{p}}{j \omega Z_{m e c h}^{E}}\right|=\left|\frac{\varphi_{m} \varphi_{p} C}{j \omega C Z_{m e c h}^{E}-\varphi_{p}^{2}}\right| .
$$

After inserting $C\left(=\frac{\varepsilon_{33}^{S} l w}{t_{p}}=\varepsilon_{33}^{S} \frac{l w}{(1-n) t_{\text {lam }}}\right)$ in the above formulae, the ME charge and voltage coefficients can be rewritten as

$$
\begin{gathered}
\alpha_{M E}^{V}=\left|\frac{d V}{d H}\right|=\frac{n d_{33, m} g_{31, p} t_{p}}{n s_{11, p}^{E}\left(1-k_{31, p}^{2}\right)+s_{33, m}^{H}(1-n)}[(\mathrm{V} / \mathrm{A}) / \mathrm{m}] \\
\alpha_{M E}^{Q}=\left|\frac{d Q}{d H}\right|=\frac{n l w d_{33, m} d_{31, p}}{n s_{11, p}^{E}+(1-n) s_{33, m}^{H}}[(\mathrm{C} / \mathrm{A}) / \mathrm{m}]
\end{gathered}
$$

\section{Charge and voltage coefficients at the resonant frequency}

From the simplified equivalent circuit in Fig. 4, the mechanical resistance can be written as $Z_{\text {mech }}^{E}=Z_{1, \text { mech }} / / Z_{1, \text { mech }}$ $+Z_{2, \text { mech }}=-\frac{j}{2} \rho v A_{\text {Lam }} \operatorname{ctg}\left(\frac{k l}{2}\right)$, and simplified using the Taylor series expansion of $\frac{\tan x}{x}=-\sum_{n} \frac{2}{x^{2}-\left(n^{2} \pi^{2} / 4\right)}(n$ is odd $)$ to

$$
Z_{\text {mech }}^{E}=-\frac{1}{2} j \rho v A\left(\frac{\frac{\pi^{2}}{4}-\frac{k^{2} l^{2}}{4}}{k l}\right)=j \omega \frac{\rho v A k l}{8 \omega}+\frac{1}{j \omega \frac{8 k l}{\rho v A \pi^{2} \omega}} .
$$

At resonance, the ME sensor can be considered as a mechanical oscillator with a wavelength, $\lambda$, equal to $2 l$, where $l$ is the length of the ME laminate. The corresponding wave number is $k=\frac{2 \pi}{\lambda}=\frac{2 \pi}{2 l}=\frac{\pi}{l}$. Thus, the equation for the mechanical impedance can be reduced to

$$
Z_{m e c h}^{E}=j \omega \frac{Z_{0} \pi}{8 \omega}+\frac{1}{j \omega \frac{8}{Z_{0} \pi \omega}}=j \omega L_{\text {mech }}+\frac{1}{j \omega C_{\text {mech }}}
$$

where $\quad L_{\text {mech }}=\frac{Z_{0} \pi}{8 \omega}, \quad C_{\text {mech }}=\frac{8}{Z_{0} \pi \omega}, \quad$ and $\quad Z_{0}=\rho v_{s} A$ $=\left(\frac{n}{s_{33, m}^{H}}+\frac{1-n}{s_{11, p}^{E}}\right) \frac{\pi A}{\omega_{s} l}$.

The equivalent mechanical resistance of the ME laminate sensor is given by ${ }^{18,19} \quad R_{\text {mech }}^{E}=\frac{\omega_{s} L_{\text {mech }}}{Q_{\text {mech }}}=\frac{\pi Z_{0}}{8 Q_{\text {mech }}}$ $=\frac{\pi}{8 Q_{\text {mech }}} \rho v A=\frac{\pi A}{8 Q_{\text {mech }} \sqrt{\rho}} \sqrt{\frac{n}{s_{33, m}^{H}}+\frac{1-n}{s_{11}^{E}}}$, where $Q_{\text {mech }}$ is the mechanical quality coefficient and $\omega_{\mathrm{s}}$ is the resonance frequency. By including the resistance (magnetic or mechanical loss) $R_{\text {mech }}$ as a dissipation, we can obtain the expression for the impedance as $Z_{\text {mech }}^{E}=R_{\text {mech }}^{E}+j \omega L_{\text {mech }}+\frac{1}{j \omega C_{\text {mech }}}$, as illustrated in the circuit of Fig. 5. Thus, we obtain the ME voltage 


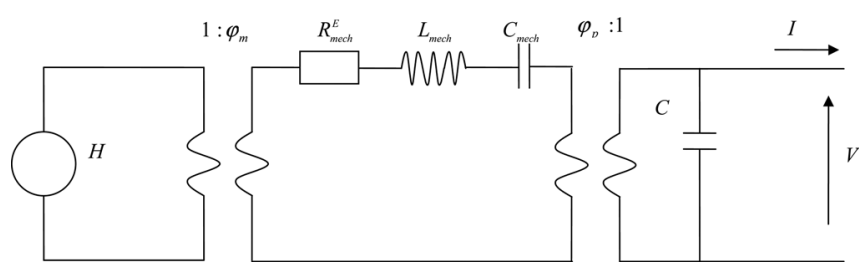

FIG. 5. Sketch view of the equivalent circuit under free boundary conditions and at the resonant frequency. $Z^{D}{ }_{\text {mech }}\left(=R_{\text {mech }}^{D}+j \omega L_{\text {mech }}+1 / j \omega C_{\text {mech }}\right)$ is the mechanical impedance at the resonant frequency.

coefficient, with the help of the simplified equivalent sensor circuit given in Fig. 8, as

$$
\alpha_{M E}^{V, f_{s}}=\left|\frac{d V}{d H}\right|=\left|\frac{\varphi_{m} \varphi_{p}}{\varphi_{p}^{2}+j \omega_{s} C\left(R_{\text {mech }}^{E}+j \omega L_{\text {mech }}+\frac{1}{j \omega C_{\text {mech }}}\right)}\right| .
$$

At $\omega^{2}=\omega_{s}^{2}=\frac{1}{L_{\text {mech }} C_{\text {mech }}}$, the value of $j \omega L_{\text {mech }}+\frac{1}{j \omega C_{\text {mech }}}=0$. We can thus reduce (11) to

$$
\alpha_{M E}^{V, f_{s}}=\left|\frac{d V}{d H}\right|=\left|\frac{\varphi_{m} \varphi_{p}}{j \omega C R_{m e c h}^{E}+\varphi_{p}^{2}}\right| .
$$

By substituting the respective expressions for $\phi_{p}, \phi_{m}, C_{0}$, and $Z_{0}$ and with the relationship $s_{11, p}^{D}=\left(1-k_{31, p}\right) s_{11, p}^{E}$, we can obtain

$$
\alpha_{M E}^{V, f_{s}}=\left|\frac{d V}{d H}\right|=\left|\frac{8 Q_{m} \frac{w t_{m} d_{33, m}}{s_{33, m}^{H}} \frac{w d_{31, p}}{s_{11, p}^{E}}}{\pi^{2} \varepsilon_{33}^{T} \frac{w}{t_{p}}\left(\frac{n}{s_{33, m}^{H}}+\frac{1-n}{s_{11, p}^{D}}\right) A}\right| .
$$

After rearrangement, the above formula becomes

$$
\alpha_{M E}^{V, f_{s}}=\left|\frac{d V}{d H}\right|_{\text {res }}=\frac{8 Q_{m}}{\pi^{2}} \frac{n t_{p} d_{33, m} g_{31, p}}{n s_{11, p}^{D}+(1-n) s_{33, m}^{H}}=\frac{8 Q_{m}}{\pi^{2}}\left|\frac{d V}{d H}\right| .
$$

Similarly, the ME charge coefficient can be obtained as

$$
\alpha_{M E}^{Q, f_{s}}=\left|\frac{d Q}{d H}\right|_{\text {res }}=\left|\frac{\varphi_{m} \varphi_{p}}{j \omega_{s}\left(R_{\text {mech }}^{E}+i \omega L_{\text {mech }}+\frac{1}{i \omega C_{\text {mech }}}\right)}\right| .
$$

After inserting $\varphi_{p}=\frac{g_{31, p}}{s_{11, p}^{D}} \frac{t_{p}}{l}, C=w \frac{d_{31, p}}{s_{11, p}^{E}}, \varphi_{m}=w t_{m} \frac{d_{33, m}}{s_{33, m}^{H}}$, and $C=\varepsilon_{33}^{S} \frac{l w}{t_{p}}$ into (14) and (15), the ME voltage and charge coefficients become

$$
\begin{aligned}
\alpha_{M E}^{V, f_{s}} & =\frac{d V}{d H}=\frac{8 Q_{m}}{\pi^{2}} \frac{n t_{p} d_{33, m} g_{31, p}}{n s_{11, p}^{D}+(1-n) s_{33, m}^{H}} \\
& =\left|\frac{8 Q_{m}}{\pi^{2}} \alpha_{M E}^{V}\right|[(\mathrm{V} / \mathrm{A}) / \mathrm{m}],
\end{aligned}
$$

$$
\begin{aligned}
\alpha_{M E}^{Q, f_{s}} & =\frac{d Q}{d H}=\frac{8 Q_{m}}{\pi^{2}} \frac{n l w d_{33, m} d_{31, p}}{n s_{11, p}^{E}+(1-n) s_{33, m}^{H}} \\
& =\left|\frac{8 Q_{m}}{\pi^{2}} \alpha_{M E}^{Q}\right|[(\mathrm{C} / \mathrm{A}) / \mathrm{m}] .
\end{aligned}
$$

\section{E. Equivalent magnetic noise at low frequencies when using a charge amplifier}

To amplify a magnetic signal sensed by the ME laminate, a charge amplifier can be used. The circuit in this case is given in Fig. 6. The Johnson noise source of the resistor $R_{l}$ is $e_{n R l}$; the equivalent noise sources of the charge amplifier $\left(e_{n}, i_{n}\right)$, and the dielectric loss factor of piezoelectric layer are considered as the main noise sources. The output voltage and equivalent magnetic noises of the sensor have been evaluated $^{22,23}$ as

$$
\begin{aligned}
e_{n, Q}^{2}(f)= & \left|Z_{1}\right|^{2}\left[i_{n \_R_{1}}^{2}(f)+i_{n}^{2}(f)+i_{n_{-} v i b}^{2}(f)\right] \\
& +\left|1+\frac{Z_{1}}{Z}\right|^{2} e_{n}^{2}(f)+\left|T_{r}(f)\right|^{2} b_{n_{-} \text {sensor }}^{2}(f)
\end{aligned}
$$

where $\quad i_{n_{-} v i b}^{2}(f)=(2 \pi f)^{2} q_{n_{-} v i b}^{2}(f), \quad Z_{1}=\frac{R_{1}}{1+j(2 \pi f) C_{1} R_{1}}$, $Z=\frac{R}{1+j(2 \pi f) C R}$, and

$$
\begin{aligned}
b_{n, Q}^{2}(f)= & \frac{1}{\alpha_{M E}^{Q}(f)}\left[\frac { 1 } { ( 2 \pi f ) ^ { 2 } } \left(i_{n}^{2}(f)+\frac{4 k_{B} T}{R_{1}}+\frac{4 k_{B} T}{R}+\frac{e_{n}^{2}(f)}{R_{1}^{2}}\right.\right. \\
& \left.\left.+\frac{e_{n}^{2}(f)}{R^{2}}\right)+\left(C_{1}+C\right)^{2} e_{n}^{2}(f)+q_{n_{-} v i b r}^{2}(f)\right] \\
& +\frac{4 k_{B} T C}{2 \pi f \alpha_{M E}^{2}(f)} \tan (\delta(f))+b_{n}^{2}(f) .
\end{aligned}
$$

The parameters $b_{n}(f)$ and $q_{n_{-} v i b}(f)$ are the magnetic noise of the sensor and the equivalent charge noise induced by mechanical vibration (i.e., elastic and/or acoustic waves), respectively. We can assume that $R \gg R_{1}$ and that the transfer function (in $\mathrm{V} / \mathrm{T}$ ) is equal to $\left(T_{r}^{Q} \approx \frac{\alpha_{M E}^{Q}(f)}{C_{1}}\right) \cdot{ }^{22,23}$ The ME charge coefficient can then be given by $\alpha_{M E}^{Q}=\frac{d Q}{d B}$ $=\frac{1}{\mu_{0}}\left(\frac{n l w d_{33, m} d_{31, p}}{n s_{11, p}^{E}+(1-n) s_{33, m}^{H}}\right)[\mathrm{C} / \mathrm{T}]$.

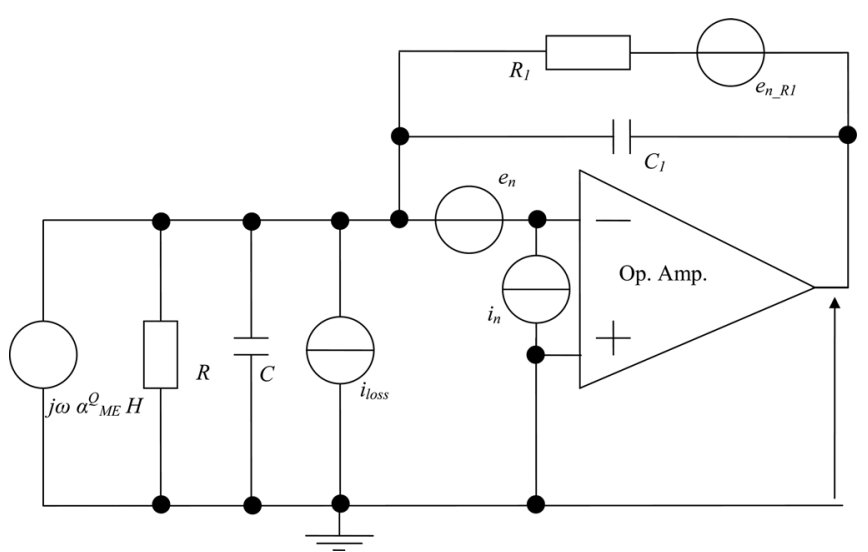

FIG. 6. Sketch view of the current charge amplifier. 
If $(R C \omega)^{2} \gg 1$ or $R \gg(C \omega)^{-1}$, and by taking strict account of the sensor parameters and noise sources of the operational amplifier, we can obtain the equivalent input magnetic noise spectral density of the sensor as

$$
\begin{aligned}
b_{n, Q}^{2}(f)= & \left(\mu_{0} \frac{n s_{11, p}^{E}+(1-n) s_{33, m}^{H}}{n l w d_{33, m} d_{31, p}}\right)^{2} \\
& \times\left[\frac{1}{(2 \pi f)^{2}}\left(i_{n}^{2}(f)+\frac{4 k_{B} T}{R}+\frac{e_{n}^{2}(f)}{R^{2}}\right)\right] \\
& +\left(\mu_{0} \frac{n s_{11, p}^{E}+(1-n) s_{33, m}^{H}}{n d_{33, m} d_{31, p}}\right)^{2} \frac{4 k_{B} T}{2 \pi f \varepsilon_{33}^{S} l w t_{p}} \tan (\delta(f)) \\
& +\left(\mu_{0} \frac{n s_{11, p}^{E}+(1-n) s_{33, m}^{H}}{n l w d_{33, m} d_{31, p}}\right)^{2} e_{n}^{2}(f) .
\end{aligned}
$$

Using a well-designed low noise amplifier and by taking into account the main sensor noise contribution, we can obtain the theoretical equivalent magnetic noise spectral density of the ME sensor unit as

$$
\begin{aligned}
b_{n, Q}(f) & =\left(\mu_{0} \frac{n s_{11, p}^{D}+(1-n) s_{33, m}^{H}\left(1-k_{31, p}^{2}\right)}{n d_{33, m} g_{31, p}}\right) \\
& \times \sqrt{\frac{i_{n}^{2}(f)}{\left(2 \pi f \varepsilon_{33}^{S} l w\right)^{2}}+\frac{e_{n}^{2}(f)}{\left(2 \pi f \varepsilon_{33}^{S} l w R\right)^{2}}+\frac{4 k_{B} T}{2 \pi f_{33}^{S} l w t_{p}} \tan (\delta(f))} \\
& \times[T / \sqrt{\mathrm{Hz}}],
\end{aligned}
$$

with an ultimate value of

$$
\begin{aligned}
b_{n, Q}(f)= & \left(\mu_{0} \frac{n s_{11, p}^{D}+(1-n) s_{33, m}^{H}\left(1-k_{31, p}^{2}\right)}{n d_{33, m} g_{31, p}}\right) \\
& \times \sqrt{\frac{4 k_{b} T}{2 \pi f \varepsilon_{33}^{S} l w t_{p}} \tan (\delta(f))[\mathrm{T} / \sqrt{\mathrm{Hz}}]} .
\end{aligned}
$$

Similarly, with the help of some classical piezoelectric and magnetostrictive parameters from Table I, we were able to evaluate the sensor noise spectral density as a function of the layer thickness ratio $n$ as

$$
\begin{aligned}
b_{n, Q}(f)= & \left(\mu_{0} \frac{n s_{11, p}^{D}+(1-n) s_{33, m}^{H}\left(1-k_{31, p}^{2}\right)}{n d_{33, m} g_{31, p}}\right) \\
& \times \sqrt{\frac{4 k_{B} T}{2 \pi f \varepsilon_{33}^{S}(1-n) V_{v o l}} \tan (\delta(f))}[\mathrm{T} / \sqrt{\mathrm{Hz}}]
\end{aligned}
$$

\begin{tabular}{|c|c|c|}
\hline Symbol & Quantity & S.I. \\
\hline$s_{33, m}^{H}$ & $\begin{array}{c}\text { Flexibility of magnetostrictive } \\
\text { layer }\end{array}$ & $40\left(\mathrm{pm}^{2} / \mathrm{N}\right)$ \\
\hline$d_{33, m}$ & Magnetostrictive constant & $11(\mathrm{~nm} / \mathrm{A})$ \\
\hline$k_{33, m}$ & $\begin{array}{l}\text { Elastic coupling factor of } \\
\text { magnetostrictive layer }\end{array}$ & 0.7 \\
\hline$s_{11, p}^{E}$ & $\begin{array}{c}\text { Flexibility of piezoelectric } \\
\text { layer }\end{array}$ & $15.9\left(\mathrm{pm}^{2} / \mathrm{N}\right)$ \\
\hline$d_{31, p}$ & Piezoelectric charge constant & $-175(\mathrm{pm} / \mathrm{V})$ \\
\hline$k_{31, p}$ & $\begin{array}{l}\text { Elastic coupling factor of } \\
\text { piezoelectric layer }\end{array}$ & 0.38 \\
\hline$g_{31, p}$ & Piezoelectric voltage constant & $-0.124(\mathrm{~V} . \mathrm{m} / \mathrm{N})$ \\
\hline$\varepsilon_{33}^{S}$ & $\begin{array}{l}\text { Dielectric coefficient under } \\
\text { constant strain }\end{array}$ & $14.113 \mathrm{nF} / \mathrm{m}$ \\
\hline $\tan (\delta(f))$ & Dielectric loss factor & $0.2 \%$ \\
\hline$l$ & Length of sensor & $80 \mathrm{~mm}$ \\
\hline$w_{\rho}$ & Width of sensor & $20 \mathrm{~mm}$ \\
\hline$t_{\text {lam }}{ }^{\mathrm{a}}$ & Thickness of sensor & $3 \mathrm{~mm}$ \\
\hline$n$ & $\begin{array}{l}\text { Relative thickness factor of } \\
\text { magnetostrictive layer }\end{array}$ & 0.8 \\
\hline
\end{tabular}

where $V_{v o l}\left(=l w t_{\text {lam }}\right)$ is the volume of the sensor. The equivalent magnetic noise spectral density as a function of $n$ is shown in Fig. 10(i) and the ME charge coefficient $\alpha_{M E}^{Q}(f)$ as a function of $n$ in Fig. 7. The analysis in term of noise shows that an optimal value of the thickness coefficient occurs for $n \approx 0.8$ contrarily to what one would expect from Fig. 7 .
TABLE I. Piezoelectric or magnetostrictive and geometrical parameters from. ${ }^{7,8}$

${ }^{\mathrm{a}} t_{\text {lam }}=t_{p}+t_{m}$

\section{F. Equivalent magnetic noise at low frequencies when using a voltage amplifier}

Similarly, the equivalent noise model for ME laminates using a voltage preamplifier detection method $^{9}$ has been analyzed following the circuit given in Fig. 8. In this case, the output voltage noise of the system can be given by

$$
\begin{aligned}
e_{n, V}^{2}(f) \approx & \left(1+\frac{R_{2}}{R_{1}}\right)^{2}\left(e_{n}^{2}+\left(R_{1} / / R_{2}\right)^{2} i_{n}^{2}+\left(\frac{R R_{x}}{R+R_{x}}\right)^{2}\right. \\
& \left.\times \frac{1}{1+\left(\frac{R R_{x}}{R+R_{x}} C \omega\right)^{2}}\left(i_{n}^{2}+i_{n \_R_{x}}^{2}+i_{n \_R}^{2}+i_{\text {loss }}^{2}\right)\right) .
\end{aligned}
$$

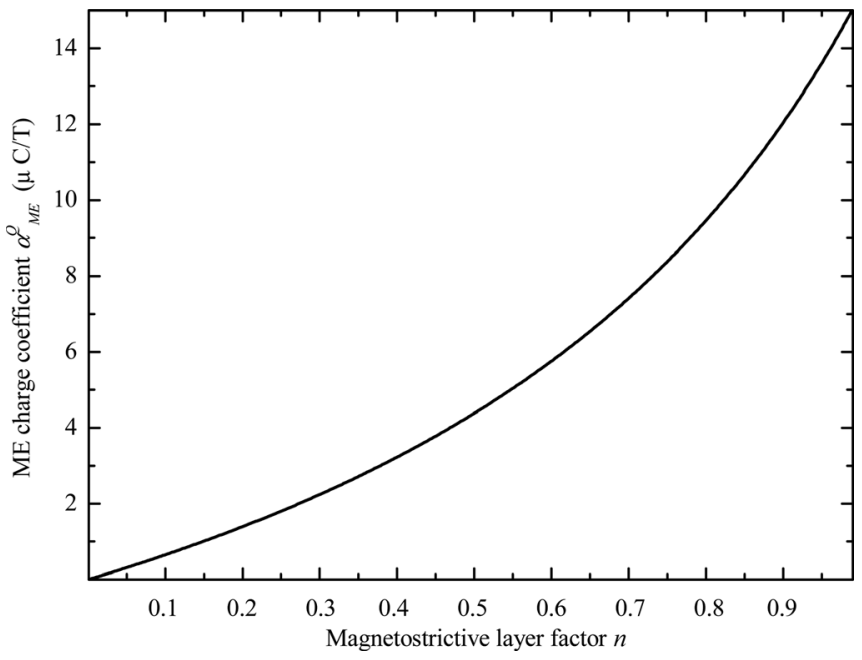

FIG. 7. Charge coefficient $\alpha_{M E}^{Q}(f)$ vs $n$ at $f=1 \mathrm{~Hz}$ with respect to data from Table I. 


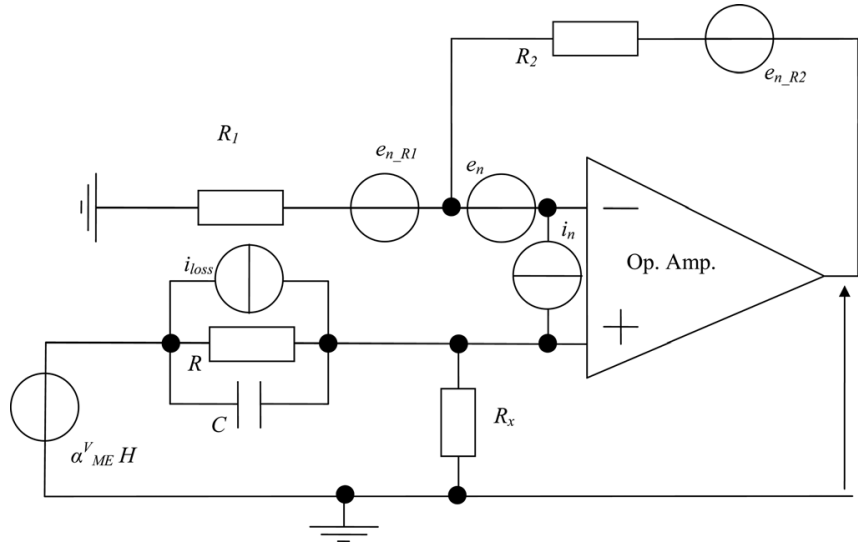

FIG. 8. Sketch view of the voltage amplifier.

We assume that $\left(R_{1} / / R_{2}\right)^{2} i_{n}^{2}$ is negligible. The output transfer function (in $V / T$ ) is

$$
T_{r}^{V}=\frac{\alpha_{M E}^{V}}{\mu_{0}}\left(1+\frac{R_{2}}{R_{1}}\right)\left(\frac{R_{x}}{R_{x}+R}\right) \frac{(1+j R C \omega)}{\left(1+j \frac{R R_{x}}{R_{x}+R} C \omega\right)} .
$$

The latter can be reduced if $R \gg R_{x}$ and $R \rightarrow \infty$ to $T_{r}^{V}=\mu_{0} \alpha_{M E}^{V}\left(1+\frac{R_{2}}{R_{1}}\right) \frac{j R_{x} C \omega}{\left(1+j R_{x} C \omega\right)}$. We can then obtain the equivalent magnetic noise spectral density of the sensor with associated voltage preamplifier electronics as

$$
\begin{aligned}
b_{n, V}^{2}(f) \approx & \left(\mu_{0} \frac{n s_{11, p}^{D}+(1-n) s_{33, m}^{H}}{n g_{31, p} d_{33, m} t_{p}}\right)^{2} \frac{1}{\left(1+(R C \omega)^{2}\right)} \\
& \times\left\{\left(\frac{\left(1+\left(\frac{R R_{x}}{R+R_{x}} C \omega\right)^{2}\right)}{\left(\frac{R_{x}}{R+R_{x}}\right)^{2}} e_{n}^{2}+R^{2}\left(i_{n}^{2}+i_{n \_R_{x}}^{2}\right.\right.\right. \\
& \left.\left.+i_{n-R_{i}}^{2}+4 k_{b} T C 2 \pi f \tan (\delta(f))\right)\right\}+b_{n}^{2}(f) .
\end{aligned}
$$

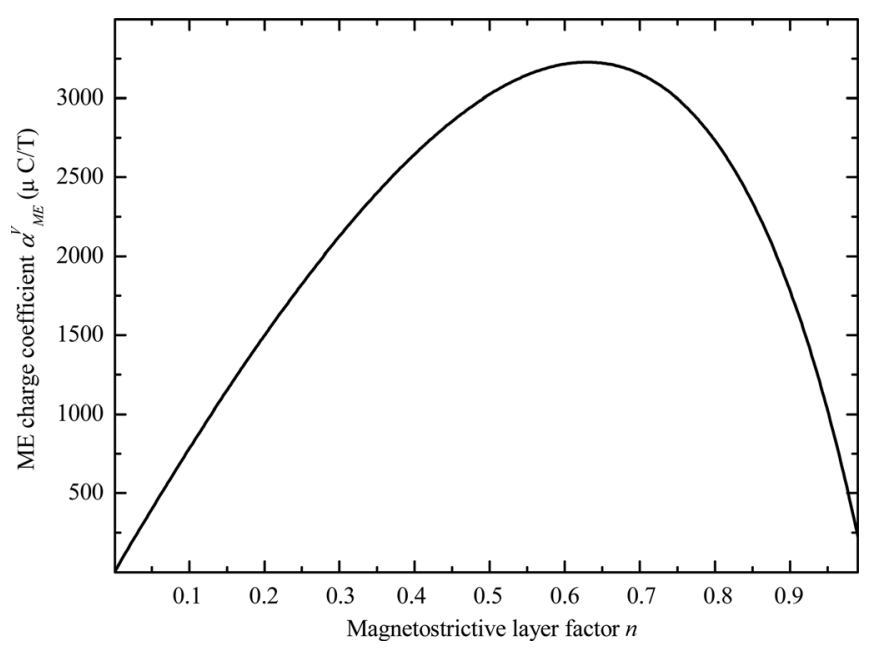

FIG. 9. Voltage coefficient $\alpha_{M E}^{V}(f)$ vs $n$ at $f=1 \mathrm{~Hz}$ with respect to data from Table I.

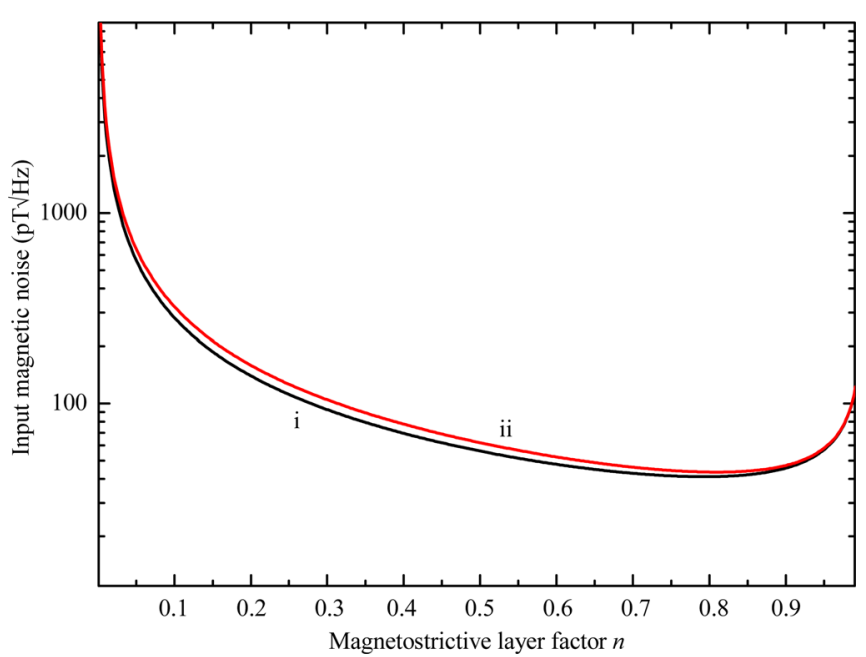

FIG. 10. (Color online) Theoretical equivalent ultimate magnetic noise $b_{n_{-} T e q}(f)$ for ME charge amplifier mode (i) and for ME voltage mode (ii) vs $n$ at $f=1 \mathrm{~Hz}$ with respect to data from Table I.

After simplification and taking account of the ME sensor parameters, the equivalent magnetic noise spectral density reduces to

$$
\begin{aligned}
b_{n, V}^{2}(f) & \approx\left(\mu_{0} \frac{n s_{11, p}^{D}+(1-n) s_{33, m}^{H}}{n g_{31, p} d_{33, m} t_{p}}\right)^{2} \\
& \times\left(\frac{R^{2} i_{n}^{2}+\frac{R^{2}}{R_{x}^{2}} e_{n}^{2}+\frac{R}{R_{x}} e_{n}^{2}+4 k_{B} T R+R^{2} 4 k_{B} T C 2 \pi f \tan (\delta(f))}{\left(1+(R C \omega)^{2}\right)}\right. \\
& \left.+e_{n}^{2}(f)\right)
\end{aligned}
$$

With a similar hypothesis as that for charge amplifiers given above (i.e., $(R C \omega)^{2} \gg 1$ or $\left.R \gg(C \omega)^{-1}\right)$, we can obtain

$$
\begin{aligned}
b_{n, V}^{2}(f) \approx & \left(\mu_{0} \frac{n s_{11, p}^{D}+(1-n) s_{33, m}^{H}}{n g_{31, p} d_{33, m} t_{p}}\right)^{2} \\
& \times\left(\frac{1}{(2 \pi f)^{2}}\left(\frac{4 k_{B} T}{R}+i_{n}^{2}+\frac{e_{n}^{2}}{R_{x}^{2}}+\frac{e_{n}^{2}}{R_{x} R}\right)\right) \\
& +\left(\mu_{0} \frac{n s_{11, p}^{D}+(1-n) s_{33, m}^{H}}{n g_{31, p} d_{33, m}}\right)^{2} \frac{4 k_{B} T}{2 \pi f \varepsilon_{33}^{S} l w t_{p}} \tan (\delta(f)) \\
& +\left(\mu_{0} \frac{n s_{11, p}^{D}+(1-n) s_{33, m}^{H}}{n g_{31, p} d_{33, m} t_{p}}\right)^{2} e_{n}^{2}+b_{n}^{2}(f) .
\end{aligned}
$$

Accordingly, the theoretical noise spectral density level in $[\mathrm{T} / \sqrt{ } / \mathrm{Hz}]$ is 
TABLE II. Theoretical charge and voltage coefficients for the four basic modes.

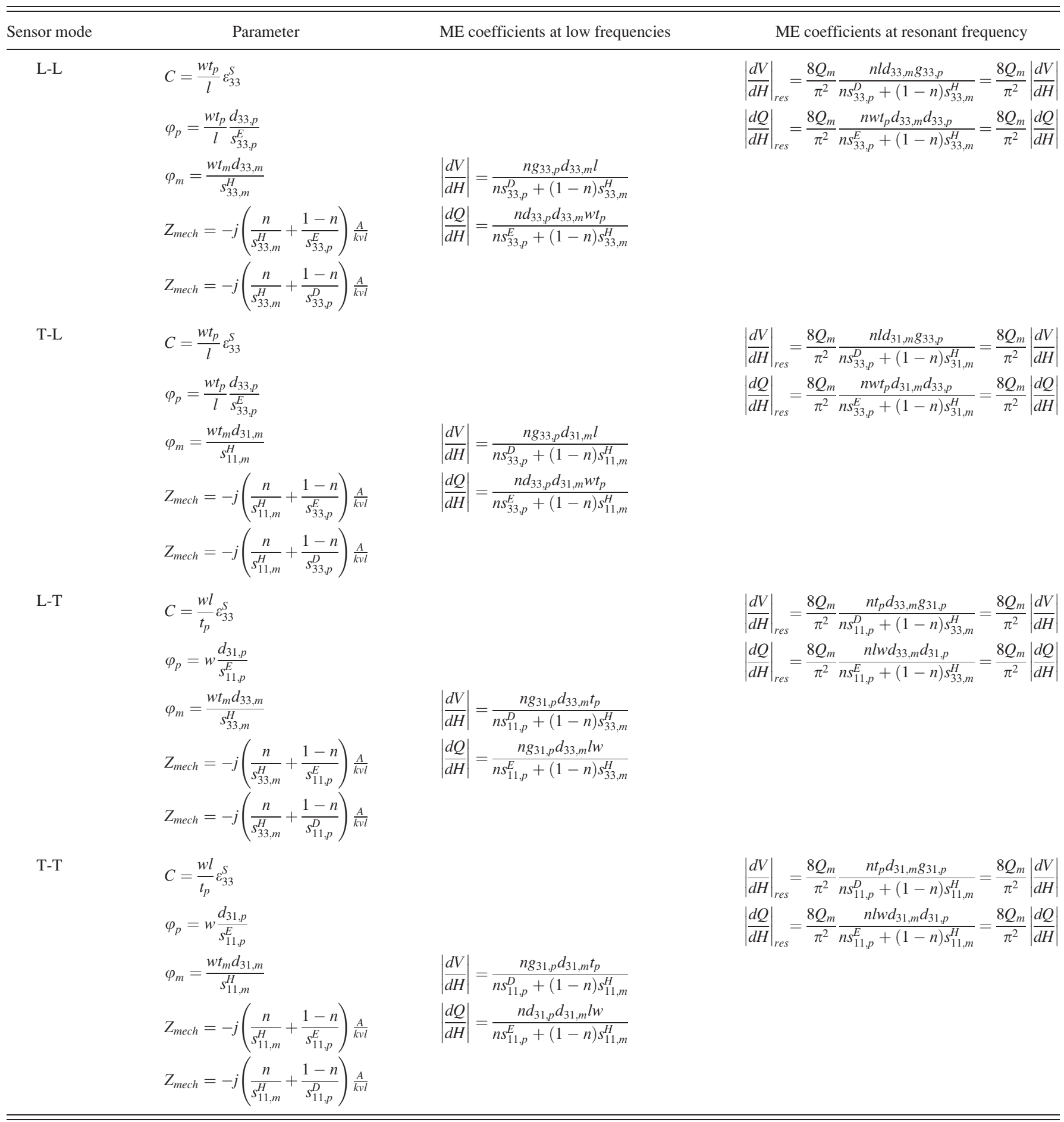

$$
b_{n, V}^{2}(f)=\left(\mu_{0} \frac{n s_{11, p}^{D}+(1-n) s_{33, m}^{H}}{n g_{31, p} d_{33, m}}\right) \sqrt{\frac{i_{n}^{2}(f)}{\left(2 \pi f \varepsilon_{33}^{S} l w\right)^{2}}+\frac{e_{n}^{2}(f)}{R_{x}^{2}\left(2 \pi f \varepsilon_{33}^{S} l w\right)^{2}}+\frac{e_{n}^{2}(f)}{R R_{x}\left(2 \pi f \varepsilon_{33}^{S} l w\right)^{2}}+\frac{4 k_{B} T}{2 \pi f \varepsilon_{33}^{S} l w t_{p}} \tan (\delta(f))},
$$

and the ultimate sensor noise spectral density is

$$
\begin{aligned}
b_{n, V}^{2}(f)= & \left(\mu_{0} \frac{n s_{11, p}^{D}+(1-n) s_{33, m}^{H}}{n g_{31, p} d_{33, m}}\right) \\
& \times \sqrt{\frac{4 k_{B} T}{2 \pi f \varepsilon_{33}^{S} l w t_{p}} \tan (\delta(f))}[\mathrm{T} / \sqrt{\mathrm{Hz}}] .
\end{aligned}
$$

Similarly, as in the previous description and by using the parameters given Table I, we can determine the ME voltage coefficient $\alpha_{M E}^{V}(f)$ (see Fig. 9) and the equivalent magnetic noise spectral density (see Fig. 10) as functions of $n$. These figures show that the optimal layer ratio factor was about $n=0.67$ for the voltage coefficient, and about $n=0.8$ for the equivalent magnetic noise spectral density. By comparing 
TABLE III. Theoretical ultimate equivalent magnetic noise of ME for the four basic modes.

\begin{tabular}{|c|c|}
\hline Sensor mode & Equivalent magnetic noise spectral density \\
\hline L-L & $b_{n}(f)=\mu_{0} \frac{n s_{33, p}^{D}+(1-n) s_{33, m}^{H}\left(1-k_{33, p}^{2}\right)}{n g_{33, p} d_{33, m}} \sqrt{\frac{4 k_{B} T}{2 \pi f V_{v o l} E_{33}^{s}} \tan (\delta(f))}$ \\
\hline $\mathrm{T}-\mathrm{L}$ & $b_{n}(f)=\mu_{0} \frac{n s_{33, p}^{D}+(1-n) s_{11, m}^{H}\left(1-k_{33, p}^{2}\right)}{n g_{33, p} d_{31, m}} \sqrt{\frac{4 k_{B} T}{2 \pi f V_{v o l} E_{33}^{S}} \tan (\delta(f))}$ \\
\hline L-T & $b_{n}(f)=\mu_{0} \frac{n s_{11, p}^{D}+(1-n) s_{3, m}^{H}\left(1-k_{31, p}^{2}\right)}{n g_{31, p} d_{33, m}} \sqrt{\frac{4 k_{B} T}{2 \pi f V_{\text {vol }} \varepsilon_{33}^{S}} \tan (\delta(f))}$ \\
\hline T-T & $b_{n}(f)=\mu_{0} \frac{\left.n{ }_{11, p}^{D}+(1-n)\right)_{1, m}^{H}\left(1-k_{31, p}^{2}\right)}{n g g_{31, p} d_{31, m}} \sqrt{\frac{4 k_{B} T}{2 \pi f V_{v o l} \varepsilon_{33}^{S}} \tan (\delta(f))}$ \\
\hline
\end{tabular}

the ultimate equivalent magnetic noise using both charge and voltage amplifiers, we can conclude that, if the circuits are well-designed, then there should be no difference between using charge and voltage amplifier detection methods for ME laminates.

\section{G. Equivalent magnetic noise at the resonant frequency when using charge or voltage amplifier}

The equivalent magnetic noise has also been investigated using the $1 \mathrm{D}$ equivalent circuit model for both charge and voltage amplifiers. Near the principal mechanical resonant frequency, a similar value for the ultimate magnetic noise spectral density was obtained for the ME laminate using either amplifier of

$$
\begin{aligned}
b_{n, f_{s}} \approx & \mu_{0} \frac{\pi^{2}}{8 Q_{m}}\left|\frac{n s_{11, p}^{D}+(1-n) s_{33, m}^{H}\left(1-k_{31, p}^{2}\right)}{n d_{33, m} g_{31, p}}\right| \\
& \times \sqrt{\frac{4 k_{B} T}{2 \pi f_{s} \varepsilon_{33}^{S} l w t_{p}} \tan (\delta(f))} .
\end{aligned}
$$

The value of $b_{n, f s}$ was estimated to be $6.5 \mathrm{fT} / \sqrt{ } \mathrm{Hz}$ at resonance using $Q_{m} \approx 50, f_{s}=30 \mathrm{kHz}$, and the data given in Table I.

\section{H. Generalization}

Finally, we extend this model to all four of the basic ME modes (L-L, T-L, L-T, T-T) using charge and voltage amplifiers. Tables II and III summarize the coefficients and the ultimate magnetic noise spectral densities that we obtained.

\section{EXPERIMENTAL MEASUREMENTS}

We have implemented both charge $\left(R_{1}=1000 \mathrm{G} \Omega\right.$, $\left.C_{l}=10 \mathrm{pF}, i_{n}=0.41 \mathrm{fA} / \sqrt{\mathrm{Hz}}\right)$ and voltage $\left(R_{l}=100 \Omega\right.$, $R_{2}=3.3 \mathrm{k} \Omega, R_{x}=10 \mathrm{G} \Omega$, and a LTC6240 OpAmp : $e_{n}=7$ $\mathrm{nV} / \sqrt{\mathrm{Hz}}, i_{n}=0.6 \mathrm{fA} / \sqrt{\mathrm{Hz}}$ ) amplifier detection methods for ME laminate sensors, using the circuits shown in Figs. 6 and 8 , respectively. The $\mathrm{ME}$ laminates consisted of $\mathrm{Pb}(\mathrm{Mg} 1 /$ $3 \mathrm{Nb} 2 / 3) \mathrm{O}_{3}-\mathrm{PbTiO}_{3}$ crystal fibers sandwiched between Metglas layers, following a structure and fabrication methods

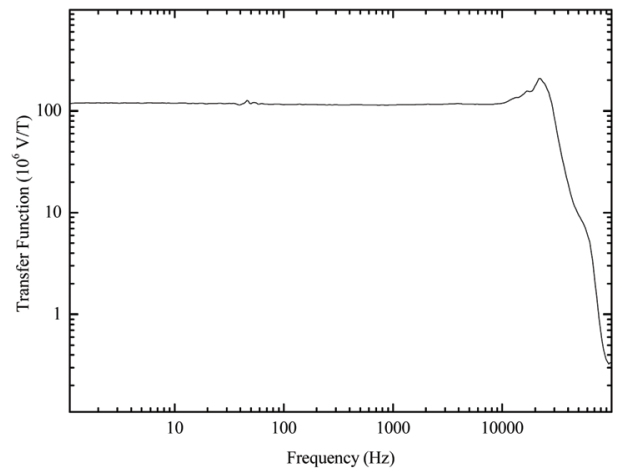

a)

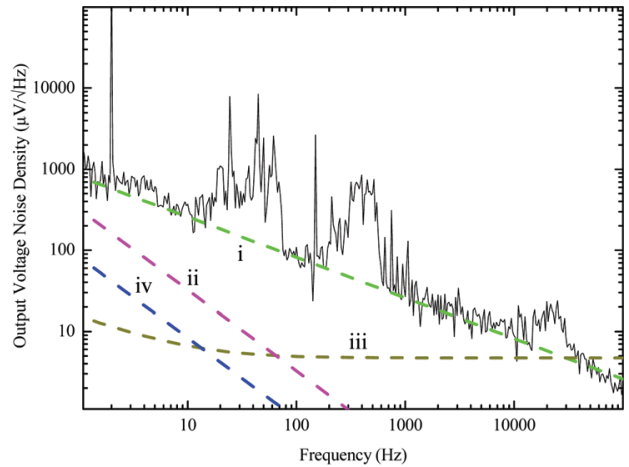

b)

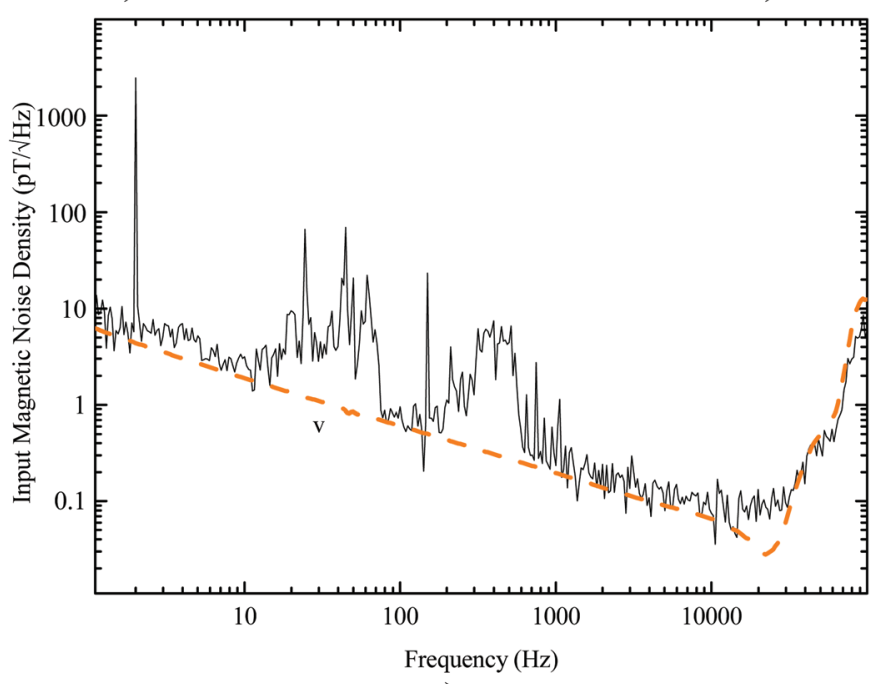

c)

FIG. 11. (Color online) (a) Magnetic transfer function, (b) output voltage noise density, and (c) equivalent input magnetic noise density for sensor F19 with an improved charge amplifier $\left(R_{l}=1 \mathrm{~T} \Omega, C_{l}=10 \mathrm{pF}\right.$, and a second stage gain of 50 ). We notice that a reference signal is used at $2 \mathrm{~Hz}$. The dashed lines indicate the theoretical noise source contributions of the dielectric loss of sensor, the current noise and voltage noise of OpAmp and the resistor $R_{1}$ (i, ii, iii \& iv, respectively). The dashed line (v) is the theoretical equivalent magnetic spectral noise density. 


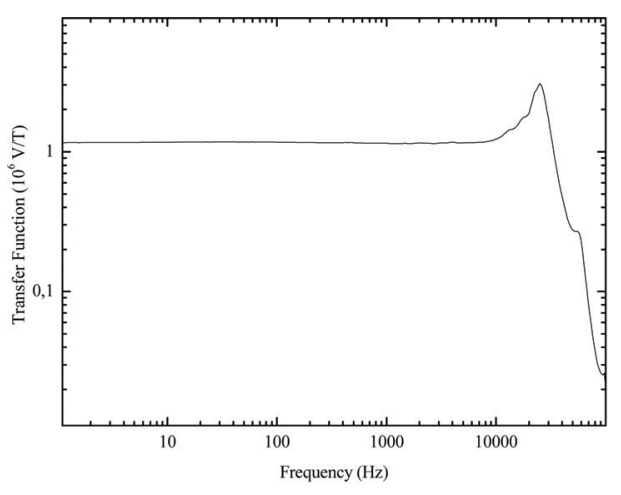

a)

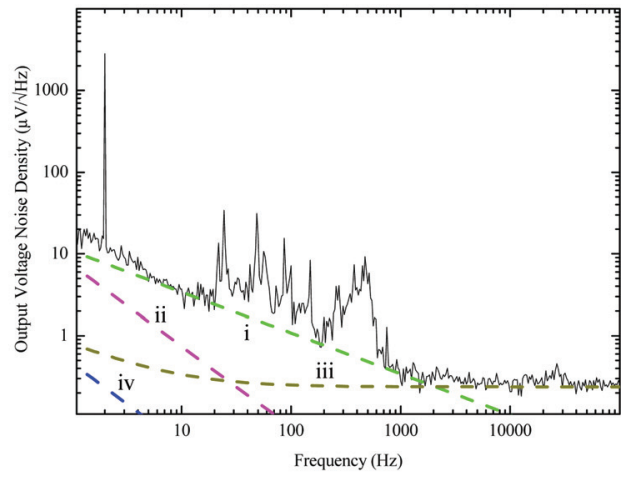

b)

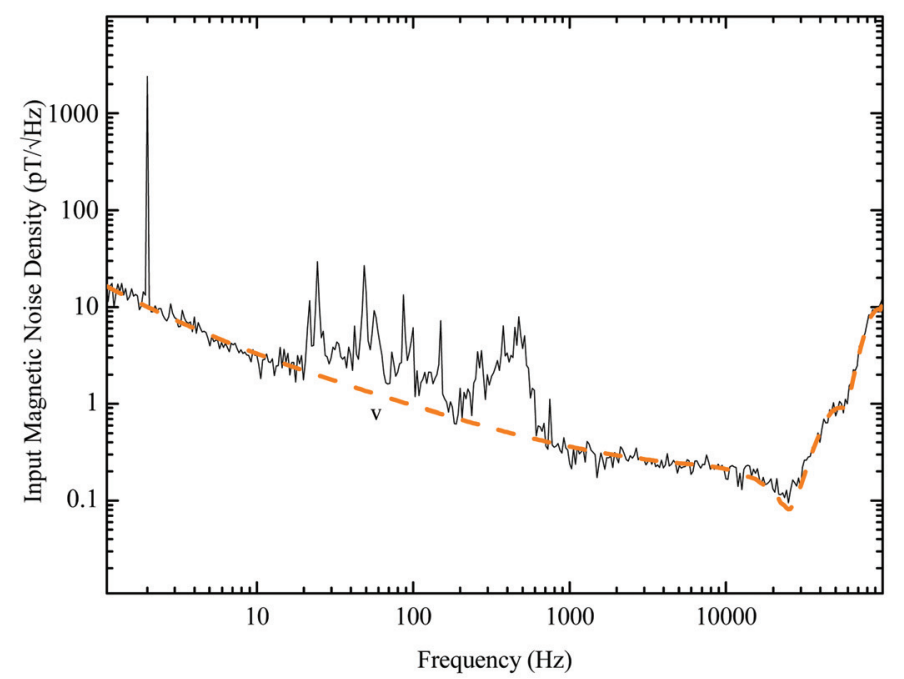

c)
FIG. 12. (Color online) (a) Magnetic transfer function, (b) output voltage noise density, and (c) equivalent input magnetic noise density for sensor F19 with a voltage amplifier $\left(R_{1}=100 \Omega\right.$, $R_{2}=3.3 \mathrm{k} \Omega, \quad R_{x}=10 \quad \mathrm{G} \Omega$, and $\mathrm{a}$ LTC6240 OpAmp). We notice that a reference signal is used at $2 \mathrm{~Hz}$. The dashed lines indicate the theoretical noise source contributions of the dielectric loss of sensor, the current noise and voltage noise of OPAmp and the resistor $R_{x}$ (i, ii, iii \& iv, respectively). The dashed line (v) is the theoretical equivalent magnetic noise density. recently reported. ${ }^{3}$ The noise sources were modeled by Eqs. (18) and (24). Their corresponding contributions to the output voltage noise are given in Figs. 11(b) and 12(b), respectively. Please note that $R_{1}$ and $R_{2}$ are both much lower than $R_{x}$, so that the effect of $R_{x}$ can be considered as negligible.

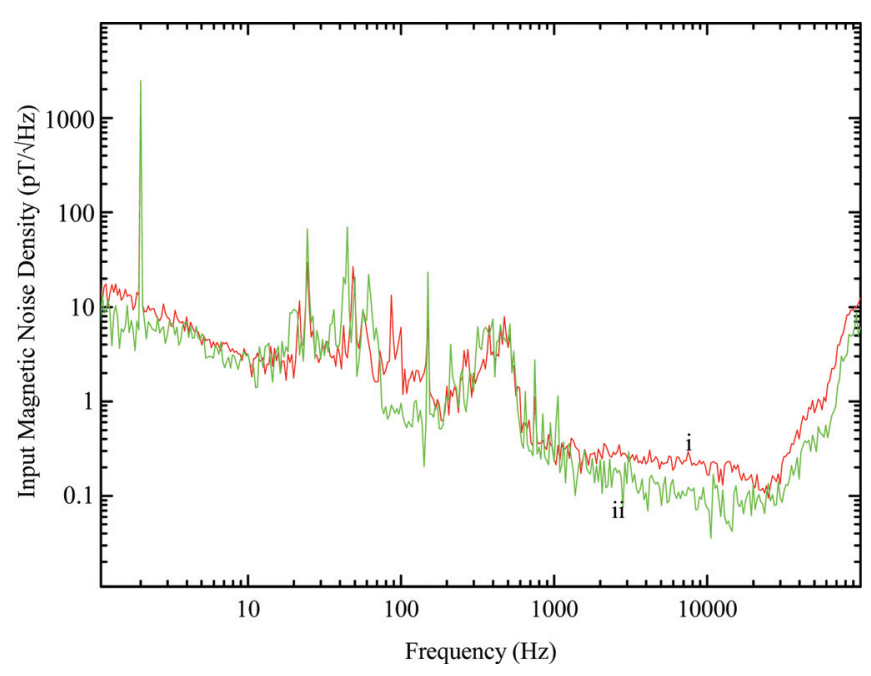

FIG. 13. (Color online) Comparison of the equivalent input magnetic noise density for sensor F19 by using (i) a voltage amplifier $\left(R_{I}=100 \Omega, R_{2}=3.3\right.$ $\mathrm{k} \Omega, R_{x}=10 \mathrm{G} \Omega$, and a LTC6240 OpAmp) or (ii) an improved charge preamplifier electronics $\left(R_{l}=1 \mathrm{~T} \Omega, C_{l}=10 \mathrm{pF}\right)$.
Using both amplification methods, the equivalent current noise of the amplifiers yielded a $1 / f$ slope in the output voltage spectral noise density, as can be seen in Fig. 12. In addition, in the middle to low frequency range, the slope of the noise density that was induced by the dielectric loss factor of the piezoelectric layer was found to vary as $1 / \sqrt{ } f$. The voltage noise of the amplifiers sets the white noise level in the frequency range of $10^{3}<f<10^{4} \mathrm{~Hz}$. Near the mechanical resonant frequency $(\approx 30 \mathrm{kHz})$, a very low equivalent magnetic noise level was obtained. The dashed orange curves in Figs. 11(c) and 12(c) show the calculated equivalent input magnetic noise and they fit quite well with the experimental results. Figure 13 compares the equivalent magnetic noise level obtained using both charge and voltage amplifiers. The results in this figure yield the same equivalent magnetic spectral noise density level as predicted by our theoretical analysis.

\section{CONCLUSION}

With the help of a 1D Mason's model, we have analyzed the equivalent magnetic noise of ME laminate sensors. Such an analysis was done for both charge and voltage amplifier detection methods. We find that the theoretical model wellpredicts the equivalent magnetic noise level of the sensor. In addition, theory suggests ways to lower the noise floor by the use of material parameters, and gives correct limits set by 
the electronic conditioning. Finally, we conclude that there are no differences in term of ME laminated sensor noise using either well-designed classical voltage or charge amplifiers. Presently, the ultimate equivalent magnetic noise of the ME sensors is limited by the volume of the laminate, and the dielectric loss factor of the piezoelectric layer(s).

\section{ACKNOWLEDGMENTS}

This work was supported by the U.S. Defense Advanced Research Projects Agency (DARPA).

${ }^{1}$ C. Nan, Phys. Rev. B 50, 6082-6088 (1994).

${ }^{2}$ L. D. Landau and E. M. Lifshitz, Electrodynamics of Continuous Media, pp. 119 (Oxford, UK, Pergamon, 1960).

${ }^{3}$ J. Das, J. Gao, Z. Xing, J. F. Li, and D. Viehland, Appl. Phys. Lett. 95, 092501 (2009).

${ }^{4}$ M. Fiebig, J. Phys. D: Appl. Phys. 38, R123-R152 (2005).

${ }^{5}$ C. Nan, M. I. Bichurin, S. Dong, D. Viehland, and G. Srinivasan, J. Appl. Phys. 103, 031101 (2008)

${ }^{6}$ J. Zhai, Z. Xing, S. Dong, J. Li, and D. Viehland, J. Am. Ceram. Soc. 91 351-358 (2008).

${ }^{7}$ S. X. Dong, J. F. Li, and D. Viehland, IEEE Trans. Ultrason. Ferroeletr. Freq. Control 50, 1253 (2003).

${ }^{8}$ S. Dong, J. F. Li, and D. Viehland, IEEE Trans. Ultrason. Ferroeletr. Freq. Control 51, 793 (2004).

${ }^{9}$ Z. Xing, J. Zhai, J. Gao, J. Li, and D. Viehland, IEEE Electron Device Lett. 30, 445 (2009).
${ }^{10}$ Z. Xing, J. Li, and D. Viehland, Appl. Phys. Lett. 91, 142905 (2007).

${ }^{11}$ L. G. C. Melo, D. Menard, A. Yelon, L. Ding, S. Saez, and C. Dolabdjian, J. Appl. Phys. 103033903 (2008).

${ }^{12}$ Z. P. Xing, J. Y. Zhai, S. X. Dong, J. F. Li, D. Viehland, and W. G. Odendaal, Meas. Sci. Technol. 19015206 (2008).

${ }^{13}$ C. D. Motchenbacher and J. A. Connelly, Low Noise Electronic System Design (Wiley, New York, 1993), Chap. 1, p. 8-18.

${ }^{14}$ G. Srinivasan, E. T. Rasmussen, B. J. Levin, and R. Hayes, Phys. Rev. B 65, 134402 (2002)

${ }^{15}$ G. Srinivasan, E. T. Rasmussen, and R. Hayes, Phys. Rev. B 67, 014418 (2003).

${ }^{16}$ S. Dong, J.F. Li, and D. Viehland, J. Appl. Phys. 95, 2625 (2004).

${ }^{17}$ S. Dong, J. Zhai, J.-F. Li, and D. Viehland, Appl. Phys. Lett. 89, 122903 (2006).

${ }^{18}$ S. Dong, J. Zhai, Chin. Sci. Bull. 53, 2113-2123 (2008).

${ }^{19}$ F. Yang, Y. M. Wen, P. Li, M. Zheng, L. X. Bian, Sens. Actuators A 141 129-135 (2008).

${ }^{20}$ D. Royer, E. Dieulesaint, Ondes élastiques dans les solides, Tome 1 (Propagation libre et guidée) p. 6-9 (Masson, Lyon, France, 1997), and Tome 2 (Génération, interaction acousto-optique, applications), p. 132-134 (Masson, Lyon, France, 1999).

${ }^{21}$ M. Brissaud, Matériaux piézoélectriques, Ch. 6, (Presses Polytechniques et Universitaires Romandes, Lyon, France, 2007).

${ }^{22}$ X. Zhuang, M. Lam Chok Sing, C. Cordier, S. Saez, and C. Dolabdjian; J. Das, J. Gao, J. F. Li and D. Viehland, "Noise analysis of Metglas/PZT fiber laminate composites as a magnetic sensor," IEEE Sens. J. (to be published).

${ }^{23}$ X. Zhuang, M. Lam Chok Sing, C. Cordier, S. Saez, and C. Dolabdjian; L. Shen, J. F. Li, M. Li, and D. Viehland, "Efficiency of applied axial field modulation technique on ME sensor input magnetic noise," IEEE Sens. J. (to be published). 